Article

\title{
Exact Solutions for a Class of Wick-Type Stochastic (3+1)-Dimensional Modified Benjamin-Bona-Mahony Equations
}

\author{
Praveen Agarwal ${ }^{1}\left(\mathbb{D}\right.$, Abd-Allah Hyder 2,3 ${ }^{(0)}$, M. Zakarya ${ }^{2,4}$, Ghada AlNemer ${ }^{5}$, \\ Clemente Cesarano ${ }^{6, *(D)}$ and Dario Assante ${ }^{7}$ (D) \\ 1 Department of Mathematics, Anand International College of Engineering, Jaipur 303012, India; \\ goyal.praveen2011@gmail.com \\ 2 Department of Mathematics, College of Science, King Khalid University, P.O. Box 9004, \\ Abha 61413, Saudi Arabia; abdallah.hyder@yahoo.com (A.-A.H.); \\ mohammed_zakaria1983@yahoo.com (M.Z.) \\ 3 Department of Engineering Mathematics and Physics, Faculty of Engineering Al-Azhar University, Cairo \\ P.O. Box 11371, Egypt \\ 4 Department of Mathematics, Faculty of Science, Al-Azhar University, Assiut P.O. Box 71524, Egypt \\ 5 Department of Mathematical Science, College of Science, Princess Nourah bint Abdulrahman University, \\ P.O. Box 105862, Riyadh 11656, Saudi Arabia; gnnemer@pnu.edu.sa \\ 6 Section of Mathematics, Università Telematica Internazionale Uninettuno, 00186 Rome, Italy \\ 7 Faculty of Engineering, Università Telematica Internazionale Uninettuno, 00186 Rome, Italy; \\ d.assante@uninettunouniversity.net \\ * Correspondence: c.cesarano@uninettunouniversity.net; Tel.: +39-06-69207675
}

Received: 9 October 2019; Accepted: 18 November 2019; Published: 3 December 2019

check for updates

\begin{abstract}
In this paper, we investigate the Wick-type stochastic (3+1)-dimensional modified Benjamin-Bona-Mahony (BBM) equations. We present a generalised version of the modified tanh-coth method. Using the generalised, modified tanh-coth method, white noise theory, and Hermite transform, we produce a new set of exact travelling wave solutions for the (3+1)-dimensional modified BBM equations. This set includes solutions of exponential, hyperbolic, and trigonometric types. With the help of inverse Hermite transform, we obtained stochastic travelling wave solutions for the Wick-type stochastic (3+1)-dimensional modified BBM equations. Eventually, by application example, we show how the stochastic solutions can be given as white noise functional solutions.
\end{abstract}

Keywords: modified BBM equations; (3+1)-dimensional equations; white noise; Brownian motion; travelling wave solutions; wick-type stochastic

MSC: $60 \mathrm{H} 15 ; 60 \mathrm{H} 35 ; 35 \mathrm{C} 07 ; 60 \mathrm{H} 40$

\section{Introduction}

In this paper, with the help of white noise theory, Hermite transform and a generalised, modified tanh-coth method, we deduce stochastic travelling wave solutions for the Wick-type stochastic (3+1)-dimensional modified BBM equations as the forms:

$$
\begin{aligned}
& U_{t}+R_{1}(t) \diamond U_{z}+R_{2}(t) \diamond U^{\diamond 2} \diamond U_{x}+R_{3}(t) \diamond U_{x y t}=0, \\
& V_{t}+R_{4}(t) \diamond V_{x}+R_{5}(t) \diamond V^{\diamond 2} \diamond V_{y}+R_{6}(t) \diamond V_{x z t}=0,
\end{aligned}
$$

and

$$
W_{t}+R_{7}(t) \diamond W_{y}+R_{8}(t) \diamond W^{\diamond 2} \diamond W_{z}+R_{9}(t) \diamond W_{x x t}=0,
$$


where $(x, y, z, t) \in \mathbb{R}^{3} \times \mathbb{R}^{+}, R_{i}(i=1,2, \ldots 9)$ are non-zero integrable functions from $\mathbb{R}^{+}$to the Kondrative distribution space $(\mathcal{S})_{-1}$, which was defined by Holden et al. in [1] as a Banach algebra with the Wick-product $\diamond$ Equations (1)-(3) are the perturbations of the (3+1)-dimensional modified BBM equations with variable coefficients:

$$
\begin{aligned}
& u_{t}+r_{1}(t) u_{z}+r_{2}(t) u^{2} u_{x}+r_{3}(t) u_{x y t}=0, \\
& v_{t}+r_{4}(t) v_{x}+r_{5}(t) v^{2} v_{y}+r_{6}(t) v_{x z t}=0
\end{aligned}
$$

and

$$
w_{t}+r_{7}(t) w_{y}+r_{8}(t) w^{2} w_{z}+r_{9}(t) w_{x x t}=0,
$$

where $r_{i}(i=1,2, \ldots 9)$ are non-zero integrable functions o $n \mathbb{R}^{+}$. The modified BBM equation:

$$
u_{t}+k(t) u_{x}+l(t) u^{2} u_{x}+m(t) u_{x x x}=0 .
$$

which describes the surface long waves in nonlinear dispersive media. It is also used as a character to acoustic-gravity waves in compressible fluids, hydromagnetic waves in cold plasma, and acoustic waves in anharmonic crystals [2]. The study of $(3+1)$-dimensional nonlinear equations is promising because these equations model the real features in a wide assortment of science, technology, fluid mechanics, wave propagations, electrodynamics, and engineering fields [3-6]. For this reason, Hereman [4,5] proposed the (3+1)-dimensional nonlinear modified KdV equation. Analogously, and by the same sense, Wazwaz [7] introduced Equations (4)-(6). Moreover, if Equations (4)-(6) are considered in a random environment, we have random (3+1)-dimensional modified BBM equations. In order to obtain the exact solutions of random (3+1)-dimensional modified BBM equations, we only consider them in a white noise environment; that is, we will discuss the Wick-type, stochastic, (3+1)-dimensional modified BBM Equations (1)-(3).

Recently, the study of solutions to nonlinear partial differential equations (PDEs) is prospering [8-10]. Many authors have researched the subject of the random travelling wave, which is a significant subject of stochastic partial differential equations (SPDEs). Wadati [11] first proposed and discussed the stochastic KdV equation and gave the propagation of soliton of the KdV equation under the effect of Gaussian noise. Furthermore, Ghany and Hyder [12-15], Ghany, Hyder and Zakarya [16,17], Chen and Xie [18-20], Hyder and Zakarya [21,22], Hyder [23,24], and Agarwal, Hyder and Zakarya [25] investigated a wide class of Wick-type stochastic evolution equations by using different extension methods and white noise analysis.

There are many methods to obtain travelling wave solutions to nonlinear PDEs, such as the inverse scattering method [26], the Newton's method [27], the tanh method [28], the Sinc-Galerkin method [29], the residual power series method [30], the semi-inverse variational principle and the first integral method [31], and the Daftardar-Gejji and Jafari method [32]. The tanh method, established by Malfliet [33], pursues a specially straightforward and effective algorithm to obtain exact solutions for a wide class of nonlinear PDEs. Moreover, a variety of research papers have focused on the different applications and extensions of the tanh method. Fan [34] has introduced an extended tanh method and gave new travelling wave solutions that cannot be obtained by the tanh method. Also, Wazwaz extended the tanh method and named it the tanh-coth method [35]. Furthermore, El-Wakil [36] and Soliman [37] modified the tanh-coth method and presented new, exact solutions for some nonlinear PDEs.

Our aim in this work was to obtain new stochastic travelling wave solutions for the Wick-type stochastic (3+1)-dimensional modified BBM equations. Firstly, we give a generalised version of the modified tanh-coth method to make it convenient for the nonlinear (3+1)-dimensional and multi dimensional PDEs. Secondly, we use the generalised, modified tanh-coth method, white noise theory, and Hermite transform to produce a new set of exact travelling wave solutions for the (3+1)-dimensional modified BBM equations, this set includes solutions of exponential, hyperbolic, and 
trigonometric types. Finally, we use the inverse Hermite transform to obtain stochastic travelling wave solutions for the Wick-type stochastic (3+1)-dimensional modified BBM equations. Moreover, by an application example, we show how the stochastic solutions can be given as white noise functional solutions. In our work, the modified BBM equation describes the surface long waves in nonlinear dispersive media. It is also used as a character of acoustic gravity waves in compressible fluids, hydromagnetic waves in cold plasma, and acoustic waves in harmonic crystals [2]. The study of $(3+1)$-dimensional nonlinear equations is prospering because these equations model the real features in a wide assortment of science, technology, fluid mechanics, wave propagations, electrodynamics, and engineering fields [3-6]. The origin and references of Equation (1) are given in Holden [1]. Ghany and Fathallah studied white-noise functional solutions for wick-type stochastic time-fractional Benjamin-Bona-Mahony (BBM) equation in [38]. Recently, Sahoo and Saha Ray studied by other methods the stochastic solutions of wick-type stochastic time-fractional BBM equation for modeling long surface gravity waves of small amplitude, in [39]. The PDE of Benjamin et al. [2] is now often called the BBM equation, although it is also known as the regularised long wave (RLW) equation. Morrison et al proposed the one-dimensional PDE, as an equally valid and accurate model for the same wave phenomena simulated by the KdV and RLW equations [40]. Random waves are an important subject of random PDEs. In essence, to investigate the exact solutions of random BBM equation, we restricted our attention to consider this problem in white noise environment [38].

This paper is organized as follows: In Section 2, we recall some requisites from Gaussian white noise analysis. In Section 3, we give a generalisation to the modified tanh-coth method to make it convenient for the nonlinear (3+1)-dimensional equations. In Section 4, we employ the generalised, modified tanh-coth method, white noise theory, and Hermite transform to obtain a new set of exact travelling wave solutions for the (3+1)-dimensional modified BBM equations. In Section 5, we apply the inverse Hermite transform to explore stochastic travelling wave solutions for the Wick-type stochastic (3+1)-dimensional modified BBM equations. In Section 5, we give some examples to show that the stochastic solutions can be given as Brownian motion functional solutions and white noise functional solutions. In Section 6, we give a summary and discussion.

\section{Requisites from Gaussian White Noise Analysis}

The Gaussian white noise analysis starts with the rigging $\mathcal{S}\left(\mathbb{R}^{d}\right) \subset L^{2}\left(\mathbb{R}^{d}\right) \subset \mathcal{S}^{*}\left(\mathbb{R}^{d}\right)$, where $\mathcal{S}\left(\mathbb{R}^{d}\right)$ is the Schwartz space of rapidly decreasing, infinite differentiable functions on $\mathbb{R}^{d}$, and $\mathcal{S}^{*}\left(\mathbb{R}^{d}\right)$ is the space of tempered distributions. From the Bochner-Minlos theorem [1], we have a unique white noise measure $\mu$, on $\left(\mathcal{S}^{*}\left(\mathbb{R}^{d}\right), \beta\left(\mathcal{S}^{*}\left(\mathbb{R}^{d}\right)\right)\right)$. Assume that $\xi_{n}(x)=\pi^{-1 / 4}((n-$ $1) !)^{-1 / 2} e^{-x^{2} / 2} h_{n-1}(\sqrt{2 x}), n \in \mathbb{N}$ are the Hermite functions, where $h_{n}(x)$ denotes the Hermite polynomials. It is well known that the collection $\left(\xi_{n}\right)_{n \in \mathbb{N}}$ forms an orthonormal basis for $L^{2}(\mathbb{R})$. Let $\alpha=\left(\alpha_{1}, \ldots, \alpha_{d}\right)$ be a $d$-dimensional multi-indices with $\alpha_{1}, \ldots, \alpha_{d} \in \mathbb{N}$; then, the family of tensor products $\xi_{\alpha}:=\xi_{\left(\alpha_{1}, \ldots, \alpha_{d}\right)}=\xi_{\alpha_{1}} \otimes \ldots \otimes \xi_{\alpha_{d}}, \alpha \in \mathbb{N}^{d}$ constitutes an orthonormal basis for $L^{2}\left(\mathbb{R}^{d}\right)$. Now, introduce an ordering in $\mathbb{N}^{d}$ by

$$
i<j \Rightarrow \sum_{k=1}^{d} \alpha_{k}^{(i)} \leq \sum_{k=1}^{d} \alpha_{k}^{(j)}, \text { where } \alpha^{(i)}=\left(\alpha_{k}^{(i)}\right)_{k=1}^{d}, \alpha^{(j)}=\left(\alpha_{k}^{(j)}\right)_{k=1}^{d} \in \mathbb{N}^{d} .
$$

Using this ordering, we define $\eta_{i}:=\xi_{\alpha^{(i)}}=\xi_{\alpha_{1}^{(i)}} \otimes \ldots \otimes \xi_{\alpha_{d}^{(i)}}, i \in \mathbb{N}$. Let $\mathbb{J}=\left(\mathbb{N}_{0}^{\mathbb{N}}\right)_{c}$ be the set of all sequences $\alpha=\left(\alpha_{i}\right)_{i \in \mathbb{N}}$ with $\alpha_{i} \in \mathbb{N}_{0}$ and with compact support. For $\alpha \in \mathbb{J}$, we define

$$
H_{\alpha}(\omega)=\prod_{i=1}^{\infty} h_{\alpha_{i}}\left(\left\langle\omega, \eta_{i}\right\rangle\right), \omega \in \mathcal{S}^{*}\left(\mathbb{R}^{d}\right)
$$


Let $n \in \mathbb{N}$, the Kondrative space of stochastic test functions $(\mathcal{S})_{1}^{n}$ is defined by:

$$
(\mathcal{S})_{1}^{n}=\left\{f=\sum_{\alpha} c_{\alpha} H_{\alpha} \in \bigoplus_{k=1}^{n} L^{2}(\mu): c_{\alpha} \in \mathbb{R}^{n} \text { and }\|f\|_{1, k}^{2}:=\sum_{\alpha} c_{\alpha}^{2}(\alpha !)^{2}(2 \mathbb{N})^{k \alpha}<\infty \forall k \in \mathbb{N}\right\},
$$

and the Kondrative space of stochastic distributions $(\mathcal{S})_{-1}^{n}$ is defined by:

$$
(\mathcal{S})_{-1}^{n}=\left\{F=\sum_{\alpha} b_{\alpha} H_{\alpha}: b_{\alpha} \in \mathbb{R}^{n} \text { and }\|F\|_{-1, k}^{2}:=\sum_{\alpha} b_{\alpha}^{2}(2 \mathbb{N})^{-q \alpha}<\infty \text { for some } q \in \mathbb{N}\right\} .
$$

The family of seminorms $\|f\|_{1, k}, k \in \mathbb{N}$ produces a topology on $(\mathcal{S})_{1}^{n}$ and $(\mathcal{S})_{-1}^{n}$ can be represented as the dual of $(\mathcal{S})_{1}^{n}$ under the action $\langle F, f\rangle=\sum_{\alpha}\left(b_{\alpha}, c_{\alpha}\right) \alpha$ !, where $F=\sum_{\alpha} b_{\alpha} H_{\alpha} \in(\mathcal{S})_{-1}^{n}, f=\sum_{\alpha} c_{\alpha} H_{\alpha} \in$ $(\mathcal{S})_{1}^{n}$ and $\left(b_{\alpha}, c_{\alpha}\right)$ is the usual scalar product on $\mathbb{R}^{n}$.

The Wick product of two distributions $F=\sum_{\alpha} a_{\alpha} H_{\alpha}, G=\sum_{\beta} b_{\beta} H_{\beta} \in(\mathcal{S})_{-1}^{n}$ with $a_{\alpha}, b_{\beta} \in \mathbb{R}^{n}$ is defined by:

$$
F \diamond G=\sum_{\alpha, \beta}\left(a_{\alpha}, b_{\beta}\right) H_{\alpha+\beta} .
$$

Let $F=\sum_{\alpha} a_{\alpha} H_{\alpha} \in(\mathcal{S})_{-1}^{n}$ with $a_{\alpha} \in \mathbb{R}^{n}$. The Hermite transform of $F$ is defined by:

$$
\mathcal{H} F(w)=\widetilde{F}(w)=\sum_{\alpha} a_{\alpha} w^{\alpha} \in \mathbb{C}^{n} \quad \text { (when convergent) }
$$

where $w=\left(w_{1}, w_{2}, \ldots\right) \in \mathbb{C}^{\mathbb{N}}$ and $w^{\alpha}=\prod_{i=1}^{\infty} w_{i}^{\alpha_{i}}$, with $\alpha=\left(\alpha_{1}, \alpha_{2}, \ldots\right) \in \mathbb{J}$ and $w_{i}^{0}=1$.

For $F, G \in(S)_{-1}^{n}$, by the definition of Hermite transform, we get:

$$
\widetilde{F \diamond G}(w)=\widetilde{F}(w) \widetilde{G}(w),
$$

for all $w$ such that $\widetilde{F}(w)$ and $\widetilde{G}(w)$ exist. The multiplication on the right hand side of the above equality is the complex bilinear multiplication in $\mathbb{C}^{n}$ which is defined by $\left(w_{1}^{1}, \ldots w_{n}^{1}\right)\left(w_{1}^{2}, \ldots, w_{n}^{2}\right)=\sum_{i=1}^{n} w_{i}^{1} w_{i}^{2}$, where $w_{i}^{k} \in \mathbb{C}$. Hence, The Hermite transform converts the Wick product into the usual product and convergence in $(\mathcal{S})_{-1}^{n}$ into pointwise and bounded convergence in a specific neighbourhood of zero in $\mathbb{C}^{n}$. For more details about stochastic Kondrative spaces, Wick product, and Hermite transform we refer the reader to [1].

\section{Generalization of the Modified Tanh-Coth Method}

Consider a multi dimensional, nonlinear PDE of wave propagation:

$$
P\left(u, u_{t}, u_{x_{i}}, u_{x_{i} x_{j}}, u_{x_{i} x_{j} x_{k}}, \ldots\right)=0,
$$

where $u$ is the dependent variable and $t=x_{0}, x_{1}, x_{2}, \ldots, x_{m}$ are the independent variables. Introduce the wave transformation:

$$
u=u(\xi), \quad \xi=\sum_{i=0}^{m} a_{i} x_{i}
$$

where $a_{i}(i=0,1,2, \ldots, m)$ are unknown constants. Therefore, Equation (15) can be transformed into a nonlinear ordinary differential equation (NODE):

$$
Q\left(u, u^{\prime}, u^{\prime \prime}, u^{\prime \prime \prime}, \ldots\right)=0 .
$$


For simplicity, we integrate the NODE (17), provided that all terms include derivatives, and set the integration constants to be zero. Subsequently, the transformed Equation (17) can be solved by expanding its general solution in finite series as follows:

$$
u(\xi)=\sum_{k=0}^{N} A_{k} \Phi^{k}(\xi)+\sum_{k=1}^{N} B_{k} \Phi^{-k}(\xi)
$$

where $\Phi$ solves the first order Riccati equation [41]:

$$
\Phi^{\prime}(\xi)=\alpha_{0}+\alpha_{1} \Phi(\xi)+\alpha_{2} \Phi^{2}(\xi),
$$

where $\alpha_{0}, \alpha_{1}$, and $\alpha_{2}$ are constants to be determined. The positive constant $N$ can be specified by balancing the linear and nonlinear terms of highest order in Equation (17). Inserting Equations (18) and (19) into Equation (17), yields an algebraic equation in $\Phi$ and its powers. Equating the coefficients of $\Phi^{k}$ to zero, gives an algebraic system of equations in $A_{k}$ and $B_{k}$. With the help of the computer symbolic system Mathematica, we can obtain $A_{k}$ and $B_{k}$. The Riccati Equation (19) has the following particular solutions [42]:

$$
\begin{cases}\Phi(\xi)=e^{\xi}-1, & \alpha_{0}=1, \alpha_{1}=1, \alpha_{2}=0, \\ \Phi(\xi)=\operatorname{coth}(\xi) \pm(\xi), \tanh (\xi) \pm i(\xi), & \alpha_{0}=\frac{1}{2}, \alpha_{1}=0, \alpha_{2}=-\frac{1}{2} \\ \Phi(\xi)=\tan (\xi),-\cot (\xi), & \alpha_{0}=1, \alpha_{1}=0, \alpha_{2}=1, \\ \Phi(\xi)=\frac{1}{2} \cot (2 \xi), \frac{1}{2} \tan (2 \xi), & \alpha_{0}=1, \alpha_{1}=0, \alpha_{2}=4 .\end{cases}
$$

\section{The Wick-Type, Stochastic, (3+1)-Dimensional Modified BBM Equations}

We first investigate the model (1) of the Wick-Type, stochastic, (3+1)-dimensional modified BBM equations. Applying Hermite transform to Equation (1), gets the deterministic equation:

$$
\begin{aligned}
\widetilde{U}_{t}(x, y, z, t, w) & +\widetilde{R}_{1}(t, w) \widetilde{U}_{z}(x, y, z, t, w)+\widetilde{R}_{2}(t, w) \widetilde{U}^{2}(x, y, z, t, w) U_{x}(x, y, z, t, w)+ \\
& +\widetilde{R}_{3}(t, w) \widetilde{U}_{x y t}(x, y, z, t, w)=0,
\end{aligned}
$$

where $w=\left(w_{1}, w_{2}, \ldots\right) \in\left(\mathbb{C}^{\mathbb{N}}\right)$. To obtain travelling wave solutions to Equation (21), we introduce the transformations $\widetilde{R_{1}}(t, w)=r_{1}(t, w), \widetilde{R_{2}}(t, w)=r_{2}(t, w), \widetilde{R_{3}}(t, w)=r_{3}(t, w)$, and $\widetilde{U}(x, y, z, t, w)=$ $u(x, y, z, t, w)=u(\xi(x, y, z, t, w))$ with

$$
\xi(x, y, z, t, w)=a_{1} x+a_{2} y+a_{3} z+b \int_{0}^{t} \chi(\tau, w) d \tau,
$$

where $a_{i}(i=1,2,3), b$, and $c$ are arbitrary constants satisfying $a_{i} b \neq 0$ and $\chi$ is a non-zero function to be determined. Hence, Equation (21) can be converted to the following NODE:

$$
\left(b \chi+a_{3} r_{1}\right) u+\frac{1}{3} a_{1} r_{2} u^{3}+a_{1} a_{2} b \chi r_{2} u^{\prime \prime}=0 .
$$

Balancing $u^{3}$ with $u^{\prime \prime}$, gives $N=1$. Therefore, we put the solution of Equation (21) in the form:

$$
u(x, y, z, t, w)=A_{0}(t, w)+A_{1}(t, w) \Phi(\xi)+\frac{B_{1}(t, w)}{\Phi(\xi)}
$$

where $\Phi$ is the solution of Equation (19). Substituting Equations (24) and (19) into Equation (23), collecting the coefficients of $\Phi^{k}(k=-3,-2,-1,0,1,2,3)$, and equating them to zero, gives the following system of seven algebraic equations in $A_{0}, A_{1}, B_{1}$, and $\chi$. 


$$
\left\{\begin{array}{l}
\left(b \chi+a_{3} r_{1}\right) A_{0}+\frac{1}{3} a_{1} r_{2} I_{0}+a_{1} a_{2} b \chi r_{3} E_{0}=0 \\
\left(b \chi+a_{3} r_{1}\right) A_{1}+\frac{1}{3} a_{1} r_{2} I_{1}+a_{1} a_{2} b \chi r_{3} E_{1}=0 \\
\left(b \chi+a_{3} r_{1}\right) B_{1}+\frac{1}{3} a_{1} r_{2} J_{1}+a_{1} a_{2} b \chi r_{3} F_{1}=0 \\
\frac{1}{3} a_{1} r_{2} I_{2}+a_{1} a_{2} b \chi r_{3} E_{2}=0 \\
\frac{1}{3} a_{1} r_{2} I_{3}+a_{1} a_{2} b \chi r_{3} E_{3}=0 \\
\frac{1}{3} a_{1} r_{2} J_{2}+a_{1} a_{2} b \chi r_{3} F_{2}=0 \\
\frac{1}{3} a_{1} r_{2} J_{3}+a_{1} a_{2} b \chi r_{3} F_{3}=0
\end{array}\right.
$$

where $I_{0}=A_{0} G_{0}+A_{1} H_{1}+B_{1} G_{1}, I_{1}=A_{0} G_{1}+A_{1} G_{0}+B_{1} G_{2}, I_{2}=A_{0} G_{2}+A_{1} G_{1}, I_{3}=A_{1} G_{2}$,

$J_{1}=A_{0} H_{1}+A_{1} H_{2}+B_{1} G_{0}, J_{2}=A_{0} H_{2}+B_{1} H_{1}, J_{3}=B_{1} H_{2}, G_{0}=A_{0}^{2}+2 A_{1} B_{1}, G_{1}=2 A_{0} A_{1}$,

$G_{2}=A_{1}^{2}, H_{1}=2 A_{0} B_{1}, H_{2}=B_{1}^{2}, E_{0}=\alpha_{0} C_{1}-\alpha_{2} D_{1}, E_{1}=\alpha_{1} C_{1}+2 \alpha_{0} C_{2}, E_{2}=\alpha_{2} C_{1}+2 \alpha_{1} C_{2}$,

$E_{3}=2 \alpha_{2} C_{2}, F_{1}=-\alpha_{1} D_{1}-2 \alpha_{2} D_{2}, F_{2}=-\alpha_{0} D_{1}-2 \alpha_{1} D_{2}, F_{3}=-2 \alpha_{0} D_{2}, C_{0}=\alpha_{0} A_{1}-\alpha_{2} B_{1}$,

$C_{1}=\alpha_{1} A_{1}, C_{2}=\alpha_{2} A_{1}, D_{1}=-\alpha_{1} B_{1}, D_{2}=-\alpha_{0} B_{1}$

Now, we solve the system (25) for some cases relating to the Riccati equation (19).

\subsection{Case I}

We reduce the system (25) by using $\alpha_{0}=\alpha_{1}=1$ and $\alpha_{2}=0$. By using Mathematica, we can find a set of solutions for the reduced system as follows:

$$
A_{0}= \pm i \sqrt{\frac{3 a_{3} r_{1}}{a_{1} r_{2}}}, \quad A_{1}=0, \quad B_{1}= \pm \sqrt{\frac{3 a_{2} a_{3} r_{1}}{a_{1} a_{2} r_{2} r_{3}-2}}, \quad \chi=\frac{2 a_{3} r_{1}}{b\left(a_{1} a_{2} r_{3}-2\right)} .
$$

Substituting the values (26) in Equation (24) and using (20), yields a travelling wave solution of Equation (21) of exponential type:

$$
\begin{aligned}
& u_{1}(x, y, z, t, w)= \\
& =\frac{ \pm i \sqrt{3 a_{3} r_{1}(t, w)\left(a_{1} a_{2} r_{2}(t, w) r_{3}(t, w)-2\right)}\left(\exp \left(\xi_{1}(x, y, z, t, w)\right)-1\right) \pm \sqrt{3 a_{1} a_{2} a_{3} r_{1}(t, w) r_{2}(t, w)}}{\sqrt{a_{1} r_{2}(t, w)\left(a_{1} a_{2} r_{2}(t, w) r_{3}(t, w)-2\right)}\left(\exp \left(\xi_{1}(x, y, z, t, w)\right)-1\right)},
\end{aligned}
$$

where

$$
\xi_{1}(x, y, z, t, w)=a_{1} x+a_{2} y+a_{3} z+2 a_{3} \int_{0}^{t} \frac{r_{1}(\tau, w)}{a_{1} a_{2} r_{3}(\tau, w)-2} d \tau
$$

\subsection{Case II}

We reduce the system (25) by using $\alpha_{0}=\frac{1}{2}, \alpha_{1}=0$, and $\alpha_{2}=-\frac{1}{2}$. By using Mathematica, we can find a set of solutions for the reduced system as follows:

$$
A_{0}=0, \quad A_{1}= \pm \sqrt{\frac{3 a_{2} a_{3} r_{1} r_{3}}{r_{2}\left(2-a_{1} a_{2} r_{3}\right)}}, \quad B_{1}= \pm i \sqrt{\frac{3 a_{1} a_{2} r_{1}}{2 r_{2} r_{3}}}, \quad \chi=-\frac{4 a_{3} r_{1}}{b\left(4+a_{1} a_{2} r_{3}\right)} .
$$


Substituting the values (29) in Equation (24) and using (20), yields travelling wave solutions of Equation (21) of hyperbolic type:

$$
\begin{aligned}
u_{2}(x, y, z, t, w)= & \pm \sqrt{\frac{3 a_{2} a_{3} r_{1}(t, w) r_{3}(t, w)}{r_{2}(t, w)\left(2-a_{1} a_{2} r_{3}(t, w)\right)}}\left(\operatorname{coth}\left(\xi_{2}(x, y, z, t, w)\right) \pm\left(\xi_{2}(x, y, z, t, w)\right)\right) \\
& \pm i \frac{\sqrt{3 b a_{2} r_{1}(t, w)}}{\sqrt{2 r_{2}(t, w) r_{3}(t, w)}\left(\operatorname{coth}\left(\xi_{2}(x, y, z, t, w)\right) \pm\left(\xi_{2}(x, y, z, t, w)\right)\right)}, \\
u_{3}(x, y, z, t, w)= & \pm \sqrt{\frac{3 a_{2} a_{3} r_{1}(t, w) r_{3}(t, w)}{r_{2}(t, w)\left(2-a_{1} a_{2} r_{3}(t, w)\right)}}\left(\tanh \left(\xi_{2}(x, y, z, t, w)\right) \pm i\left(\xi_{2}(x, y, z, t, w)\right)\right) \\
& \pm i \frac{\sqrt{3 b a_{2} r_{1}(t, w)}}{\sqrt{2 r_{2}(t, w) r_{3}(t, w)}\left(\tanh \left(\xi_{2}(x, y, z, t, w)\right) \pm i\left(\xi_{2}(x, y, z, t, w)\right)\right)},
\end{aligned}
$$

where

$$
\xi_{2}(x, y, z, t, w)=a_{1} x+a_{2} y+a_{3} z-4 a_{3} \int_{0}^{t} \frac{r_{1}(\tau, w)}{4+a_{1} a_{2} r_{3}(\tau, w)} d \tau
$$

\subsection{Case III}

We reduce the system (25) by putting $\alpha_{0}=\alpha_{2}=1$ and $\alpha_{1}=0$. By using Mathematica, we can find a set of solutions for the reduced system as follows:

$$
A_{0}= \pm \sqrt{\frac{3 a_{2} a_{3} r_{1}}{1-a_{1} a_{2} r_{2} r_{3}}}, \quad A_{1}=B_{1}= \pm \sqrt{\frac{6 a_{2} a_{3} r_{1} r_{3}}{1+2 a_{1} a_{2} r_{2} r_{3}}}, \quad \chi=\frac{-a_{3} r_{1}}{b\left(1+2 a_{1} a_{2} r_{3}\right)} .
$$

Substituting the values (33) in Equation (24) and using (20), yields travelling wave solutions of Equation (21) of trigonometric type:

$$
\begin{aligned}
& u_{4}(x, y, z, t, w)=u_{5}(x, y, z, t, w)= \\
& = \pm \sqrt{\frac{3 a_{2} a_{3} r_{1}(t, w)}{1-a_{1} a_{2} r_{2}(t, w) r_{3}(t, w)}} \pm \sqrt{\frac{6 a_{2} a_{3} r_{1}(t, w) r_{3}(t, w)}{1+2 a_{1} a_{2} r_{2}(t, w) r_{3}(t, w)}}\left(\sec \left(\xi_{3}(x, y, z, t, w)\right) \csc \left(\xi_{3}(x, y, z, t, w)\right)\right)
\end{aligned}
$$

where

$$
\xi_{3}(x, y, z, t, w)=a_{1} x+a_{2} y+a_{3} z-a_{3} \int_{0}^{t} \frac{r_{1}(\tau, w)}{1+2 a_{1} a_{2} r_{3}(\tau, w)} d \tau
$$

\subsection{Case IV}

We reduce the system (25) by putting $\alpha_{0}=1, \alpha_{1}=0$ and $\alpha_{2}=4$. By using Mathematica, we can find a set of solutions for the reduced system as follows:

$$
A_{0}= \pm 3 \sqrt{\frac{3 a_{2} a_{3} r_{1}}{9 a_{1} a_{2} r_{2} r_{3}-2}}, \quad A_{1}= \pm 8 \sqrt{\frac{3 a_{2} a_{3} r_{1} r_{3}}{2+15 a_{1} a_{2} r_{2} r_{3}}}, \quad B_{1}= \pm i \sqrt{\frac{3 a_{3} r_{1}}{a_{1} r_{2} r_{3}}}, \quad \chi=-\frac{2 a_{3} r_{1}}{b\left(9 a_{1} a_{2} r_{2} r_{3}-2\right)}
$$

Substituting the values (36) in Equation (24) and using (20), yields travelling wave solutions of Equation (21) of trigonometric type:

$$
\begin{aligned}
u_{6}(x, y, z, t, w)= \pm & 3 \sqrt{\frac{3 a_{2} a_{3} r_{1}(t, w)}{9 a_{1} a_{2} r_{2}(t, w) r_{3}(t, w)-2}} \pm 4 \sqrt{\frac{3 a_{2} a_{3} r_{1}(t, w) r_{3}(t, w)}{2+15 a_{1} a_{2} r_{2}(t, w) r_{3}(t, w)}}\left(\cot \left(2 \xi_{4}(x, y, z, t, w)\right)\right. \\
& \pm i \frac{\sqrt{3 a_{3} r_{1}(t, w)}}{2 \sqrt{a_{1} r_{2}(t, w) r_{3}(t, w)}\left(\cot \left(2 \xi_{4}(x, y, z, t, w)\right)\right)}
\end{aligned}
$$




$$
\begin{aligned}
u_{7}(x, y, z, t, w)= & \pm 3 \sqrt{\frac{3 a_{2} a_{3} r_{1}(t, w)}{9 a_{1} a_{2} r_{2}(t, w) r_{3}(t, w)-2}} \pm 4 \sqrt{\frac{3 a_{2} a_{3} r_{1}(t, w) r_{3}(t, w)}{2+15 a_{1} a_{2} r_{2}(t, w) r_{3}(t, w)}}\left(\tan \left(2 \xi_{4}(x, y, z, t, w)\right)\right. \\
& \pm i \frac{\sqrt{3 a_{3} r_{1}(t, w)}}{2 \sqrt{a_{1} r_{2}(t, w) r_{3}(t, w)}\left(\tan \left(2 \xi_{4}(x, y, z, t, w)\right)\right)}
\end{aligned}
$$

where

$$
\xi_{4}(x, y, z, t, w)=a_{1} x+a_{2} y+a_{3} z+2 a_{3} \int_{0}^{t} \frac{r_{1}(\tau, w)}{9 a_{1} a_{2} r_{3}(\tau, w)-2} d \tau .
$$

Obviously, there are several particular solutions for the system (25) with the Riccati equation (19), coming from many different cases. In the above cases we just clarified how far our technique is applicable.

Now, for $q<\infty, r>0$, consider the infinite dimensional neighbourhoods $K_{q}(r)=\left\{\left(w_{1}, w_{2}, \ldots\right) \in\right.$ $\left.\mathbb{C}^{\mathbb{N}}: \sum_{\alpha \neq 0}\left|w^{\alpha}\right|^{2}(2 \mathbb{N})^{q \alpha}<r^{2}\right\}$ of zero in $\mathbb{C}^{\mathbb{N}}$ [1]. The properties of exponential, hyperbolic, and trigonometric functions yield that there exists a bounded open set $\mathbf{D} \subset \mathbb{R}^{3} \times \mathbb{R}_{+}, q<\infty, r>0$, such that the solution $u(x, y, z, t, w)$ of Equation (21) and all its derivatives which are involved in Equation (21) are uniformly bounded for $(x, y, z, t, w) \in \mathbf{D} \times K_{q}(r)$, continuous with respect to $(x, y, z, t) \in \mathbf{D}$ for all $w \in K_{q}(r)$ and analytic with respect to $w \in K_{q}(r)$, for all $(x, y, z, t) \in \mathbf{D}$. From Theorem 4.1.1 in [1], there exists $U(x, y, z, t) \in(\mathcal{S})_{-1}$ such that $u(x, y, z, t, w)=\widetilde{U}(x, y, z)(w)$ for all $(x, y, z, t, w) \in \mathbf{D} \times K_{q}(r)$ and $U(x, y, z, t)$ solves Equation (1) in $(\mathcal{S})_{-1}$. Hence, by applying the inverse Hermite transform to Equations (27), (30), (31), (34), (37), and (38), we obtain the solutions of Equation (1) as follows:

(I) Stochastic Travelling Wave Solution of Exponential Type:

$$
\begin{aligned}
& U_{1}(x, y, z, t)= \\
& \frac{ \pm i \sqrt{3 a_{3} R_{1}(t) \diamond\left(a_{1} a_{2} R_{2}(t) \diamond R_{3}(t)-2\right)} \diamond\left(\exp ^{\diamond}\left(\Xi_{1}(x, y, z, t)\right)-1\right) \pm \sqrt{3 a_{1} a_{2} a_{3} R_{1}(t) \diamond R_{2}(t)}}{\sqrt{a_{1} R_{2}(t) \diamond\left(a_{1} a_{2} R_{2}(t) \diamond R_{3}(t)-2\right)}\left(\exp ^{\diamond}\left(\Xi_{1}(x, y, z, t)\right)-1\right)},
\end{aligned}
$$

with

$$
\Xi_{1}(x, y, z, t)=a_{1} x+a_{2} y+a_{3} z+2 a_{3} \int_{0}^{t} \frac{R_{1}(\tau)}{a_{1} a_{2} R_{3}(\tau)-2} d \tau .
$$

(II) Stochastic Travelling Wave Solutions of Hyperbolic Type:

$$
\begin{aligned}
U_{2}(x, y, z, t)= & \pm \sqrt{\frac{3 a_{2} a_{3} R_{1}(t) \diamond R_{3}(t)}{R_{2}(t) \diamond\left(2-a_{1} a_{2} R_{3}(t)\right)}} \diamond\left(\operatorname{coth}^{\diamond}\left(\Xi_{2}(x, y, z, t)\right) \pm^{\diamond}\left(\Xi_{2}(x, y, z, t)\right)\right) \\
& \pm i \frac{\sqrt{3 b a_{2} R_{1}(t)}}{\sqrt{2 R_{2}(t) \diamond R_{3}(t) \diamond\left(\operatorname{coth}^{\diamond}\left(\Xi_{2}(x, y, z, t)\right) \pm \diamond\left(\Xi_{2}(x, y, z, t)\right)\right)}} \\
U_{3}(x, y, z, t)= & \pm \sqrt{\frac{3 a_{2} a_{3} R_{1}(t) \diamond R_{3}(t)}{R_{2}(t) \diamond\left(2-a_{1} a_{2} R_{3}(t)\right)}} \diamond\left(\tanh ^{\diamond}\left(\Xi_{2}(x, y, z, t)\right) \pm i^{\diamond}\left(\Xi_{2}(x, y, z, t)\right)\right) \\
& \pm i \frac{\sqrt{3 b a_{2} R_{1}(t)}}{\sqrt{2 R_{2}(t) \diamond R_{3}(t) \diamond\left(\tanh ^{\diamond}\left(\Xi_{2}(x, y, z, t)\right) \pm i \diamond\left(\Xi_{2}(x, y, z, t)\right)\right)}}
\end{aligned}
$$

with

$$
\Xi_{2}(x, y, z, t)=a_{1} x+a_{2} y+a_{3} z-4 a_{3} \int_{0}^{t} \frac{R_{1}(\tau)}{4+a_{1} a_{2} R_{3}(\tau)} d \tau
$$


(III) Stochastic Travelling Wave Solutions of Trigonometric Type:

$$
\begin{aligned}
U_{4}(x, y, z, t)=U_{5}(x, y, z, t)= & \pm \sqrt{\frac{3 a_{2} a_{3} R_{1}(t)}{1-a_{1} a_{2} R_{2}(t) \diamond R_{3}(t)}} \pm \sqrt{\frac{6 a_{2} a_{3} R_{1}(t) \diamond R_{3}(t)}{1+2 a_{1} a_{2} R_{2}(t) \diamond R_{3}(t)}} \\
& \diamond\left(\sec ^{\diamond}\left(\Xi_{3}(x, y, z, t)\right) \diamond \csc ^{\diamond}\left(\Xi_{3}(x, y, z, t)\right)\right),
\end{aligned}
$$

with

$$
\begin{aligned}
& \Xi_{3}(x, y, z, t)=a_{1} x+a_{2} y+a_{3} z-a_{3} \int_{0}^{t} \frac{R_{1}(\tau)}{1+2 a_{1} a_{2} R_{3}(\tau)} d \tau \\
U_{6}(x, y, z, t)= & \pm 3 \sqrt{\frac{3 a_{2} a_{3} R_{1}(t)}{9 a_{1} a_{2} R_{2}(t) \diamond R_{3}(t)-2}} \pm 4 \sqrt{\frac{3 a_{2} a_{3} R_{1}(t) \diamond R_{3}(t)}{2+15 a_{1} a_{2} R_{2}(t) \diamond R_{3}(t)}} \diamond\left(\cot ^{\diamond}\left(2 \Xi_{4}(x, y, z, t)\right)\right. \\
& \pm i \frac{\sqrt{3 a_{3} R_{1}(t)}}{2 \sqrt{a_{1} R_{2}(t) \diamond R_{3}(t) \diamond\left(\cot ^{\diamond}\left(2 \Xi_{4}(x, y, z, t)\right)\right.}} \\
U_{7}(x, y, z, t)= & \pm 3 \sqrt{\frac{3 a_{2} a_{3} R_{1}(t)}{9 a_{1} a_{2} R_{2}(t) \diamond R_{3}(t)-2}} \pm 4 \sqrt{\frac{3 a_{2} a_{3} R_{1}(t) \diamond R_{3}(t)}{2+15 a_{1} a_{2} R_{2}(t) \diamond R_{3}(t)}} \diamond\left(\tan ^{\diamond}\left(2 \Xi_{4}(x, y, z, t)\right)\right) \\
& \pm i \frac{\sqrt{3 a_{3} R_{1}(t)}}{2 \sqrt{a_{1} R_{2}(t) \diamond R_{3}(t) \diamond\left(\tan ^{\diamond}\left(2 \Xi_{4}(x, y, z, t)\right)\right)}}
\end{aligned}
$$

with

$$
\Xi_{4}(x, y, z, t)=a_{1} x+a_{2} y+a_{3} z+2 a_{3} \int_{0}^{t} \frac{R_{1}(\tau)}{9 a_{1} a_{2} R_{3}(\tau)-2} d \tau .
$$

For the other two forms of the Wick-type, stochastic, $(3+1)$-dimensional modified BBM equations (2) and (3), we can follow the same technique as presented for the first form (1). Therefore, we just list the stochastic travelling wave solutions for each form. For Equation (2) one obtains the following stochastic travelling wave solution:

(I) Stochastic Travelling Wave Solution of Exponential Type:

$$
\begin{aligned}
& V_{1}(x, y, z, t)= \\
& \frac{ \pm i \sqrt{3 b_{3} R_{4}(t) \diamond\left(b_{1} b_{2} R_{5}(t) \diamond R_{6}(t)-2\right) \diamond}\left(\exp ^{\diamond}\left(\Lambda_{1}(x, y, z, t)\right)-1\right) \pm \sqrt{3 b_{1} b_{2} b_{3} R_{4}(t) \diamond R_{5}(t)}}{\sqrt{b_{1} R_{5}(t) \diamond\left(b_{1} b_{2} R_{5}(t) \diamond R_{6}(t)-2\right)}},
\end{aligned}
$$

with

$$
\Lambda_{1}(x, y, z, t)=b_{1} x+b_{2} y+b_{3} z+2 b_{3} \int_{0}^{t} \frac{R_{4}(\tau)}{b_{1} b_{2} R_{6}(\tau)-2} d \tau
$$

(II) Stochastic Travelling Wave Solutions of Hyperbolic Type:

$$
\begin{aligned}
V_{2}(x, y, z, t)= & \pm \sqrt{\frac{3 b_{2} b_{3} R_{4}(t) \diamond R_{6}(t)}{R_{5}(t) \diamond\left(2-b_{1} b_{2} R_{6}(t)\right)}} \diamond\left(\operatorname{coth}^{\diamond}\left(\Lambda_{2}(x, y, z, t)\right) \pm^{\diamond}\left(\Lambda_{2}(x, y, z, t)\right)\right) \\
& \pm i \frac{\sqrt{3 b^{*} b_{2} R_{4}(t)}}{\sqrt{2 R_{5}(t) \diamond R_{6}(t) \diamond\left(\operatorname{coth}^{\diamond}\left(\Lambda_{2}(x, y, z, t)\right) \pm \diamond\left(\Lambda_{2}(x, y, z, t)\right)\right)}} \\
V_{3}(x, y, z, t)= & \pm \sqrt{\frac{3 b_{2} b_{3} R_{4}(t) \diamond R_{6}(t)}{R_{5}(t) \diamond\left(2-b_{1} b_{2} R_{6}(t)\right)}} \diamond\left(\tanh ^{\diamond}\left(\Lambda_{2}(x, y, z, t)\right) \pm i^{\diamond}\left(\Lambda_{2}(x, y, z, t)\right)\right) \\
& \pm i \frac{\sqrt{3 b^{*} b_{2} R_{4}(t)}}{\sqrt{2 R_{5}(t) \diamond R_{6}(t)} \diamond\left(\tanh ^{\diamond}\left(\Lambda_{2}(x, y, z, t)\right) \pm i \diamond\left(\Lambda_{2}(x, y, z, t)\right)\right)}
\end{aligned}
$$


with

$$
\Lambda_{2}(x, y, z, t)=b_{1} x+b_{2} y+b_{3} z-4 b_{3} \int_{0}^{t} \frac{R_{4}(\tau)}{4+b_{1} b_{2} R_{6}(\tau)} d \tau .
$$

(III) Stochastic Travelling Wave Solutions of Trigonometric Type:

$$
\begin{aligned}
V_{4}(x, y, z, t)=V_{5}(x, y, z, t)= & \pm \sqrt{\frac{3 b_{2} b_{3} R_{4}(t)}{1-b_{1} b_{2} R_{5}(t) \diamond R_{6}(t)}} \pm \sqrt{\frac{6 b_{2} b_{3} R_{4}(t) \diamond R_{6}(t)}{1+2 b_{1} b_{2} R_{5}(t) \diamond R_{6}(t)}} \\
& \diamond\left(\sec ^{\diamond}\left(\Lambda_{3}(x, y, z, t)\right) \diamond \csc ^{\diamond}\left(\Lambda_{3}(x, y, z, t)\right)\right),
\end{aligned}
$$

with

$$
\begin{aligned}
& \Lambda_{3}(x, y, z, t)=b_{1} x+b_{2} y+a_{3} z-b_{3} \int_{0}^{t} \frac{R_{4}(\tau)}{1+2 b_{1} b_{2} R_{6}(\tau)} d \tau \\
& V_{6}(x, y, z, t)= \pm 3 \sqrt{\frac{3 b_{2} b_{3} R_{4}(t)}{9 b_{1} b_{2} R_{5}(t) \diamond R_{6}(t)-2}} \pm 4 \sqrt{\frac{3 b_{2} b_{3} R_{4}(t) \diamond R_{6}(t)}{2+15 b_{1} b_{2} R_{5}(t) \diamond R_{6}(t)}} \diamond\left(\cot ^{\diamond}\left(2 \Lambda_{4}(x, y, z, t)\right)\right. \\
& \pm i \frac{\sqrt{3 b_{3} R_{4}(t)}}{2 \sqrt{b_{1} R_{5}(t) \diamond R_{6}(t) \diamond\left(\cot ^{\diamond}\left(2 \Lambda_{4}(x, y, z, t)\right)\right)}} \\
& V_{7}(x, y, z, t)= \pm 3 \sqrt{\frac{3 b_{2} b_{3} R_{4}(t)}{9 b_{1} b_{2} R_{5}(t) \diamond R_{6}(t)-2}} \pm 4 \sqrt{\frac{3 b_{2} b_{3} R_{4}(t) \diamond R_{6}(t)}{2+15 b_{1} b_{2} R_{5}(t) \diamond R_{6}(t)}} \diamond\left(\tan ^{\diamond}\left(2 \Lambda_{4}(x, y, z, t)\right)\right. \\
& \pm i \frac{\sqrt{3 b_{3} R_{4}(t)}}{2 \sqrt{b_{1} R_{5}(t) \diamond R_{6}(t) \diamond\left(\tan ^{\diamond}\left(2 \Lambda_{4}(x, y, z, t)\right)\right)}}
\end{aligned}
$$

with

$$
\Lambda_{4}(x, y, z, t)=b_{1} x+b_{2} y+b_{3} z+2 b_{3} \int_{0}^{t} \frac{R_{4}(\tau)}{9 b_{1} b_{2} R_{6}(\tau)-2} d \tau,
$$

where $b_{i}(i=1,2,3)$ and $b^{*}$ are arbitrary constants satisfying $b_{i} b \neq 0$.

For Equation (3) one obtains the following stochastic travelling wave solution:

(I) Stochastic Travelling Wave Solution of Exponential Type:

$$
\begin{aligned}
& W_{1}(x, y, z, t)= \\
& \frac{ \pm i \sqrt{3 c_{1} R_{7}(t) \diamond\left(c_{1} c_{2} R_{8}(t) \operatorname{diamond}_{9}(t)-2\right)} \diamond\left(\exp ^{\diamond}\left(\Delta_{1}(x, y, z, t)\right)-1\right) \pm \sqrt{3 c_{1}^{2} c_{2} R_{7}(t) \diamond R_{8}(t)}}{\sqrt{c_{1} R_{8}(t) \diamond\left(c_{1} c_{2} R_{8}(t) \diamond R_{9}(t)-2\right)}\left(\exp ^{\diamond}\left(\Delta_{1}(x, y, z, t)\right)-1\right)},
\end{aligned}
$$

with

$$
\Delta_{1}(x, y, z, t)=c_{1} x+c_{2} y+c_{3} z+2 c_{1} \int_{0}^{t} \frac{R_{7}(\tau)}{c_{1} c_{2} R_{9}(\tau)-2} d \tau .
$$

(II) Stochastic Travelling Wave Solutions of Hyperbolic Type:

$$
\begin{aligned}
W_{2}(x, y, z, t)= & \pm \sqrt{\frac{3 c_{1} c_{2} R_{7}(t) \diamond R_{9}(t)}{R_{8}(t) \diamond\left(2-c_{1} c_{2} R_{9}(t)\right)}} \diamond\left(\operatorname{coth}^{\diamond}\left(\Delta_{2}(x, y, z, t)\right) \pm^{\diamond}\left(\Delta_{2}(x, y, z, t)\right)\right) \\
& \pm i \frac{\sqrt{3 b^{* *} c_{2} R_{7}(t)}}{\sqrt{2 R_{8}(t) \diamond R_{9}(t)} \diamond\left(\operatorname{coth}^{\diamond}\left(\Delta_{2}(x, y, z, t)\right) \pm \diamond\left(\Delta_{2}(x, y, z, t)\right)\right)}
\end{aligned}
$$




$$
\begin{aligned}
W_{3}(x, y, z, t)= & \pm \sqrt{\frac{3 c_{1} c_{2} R_{7}(t) \diamond R_{9}(t)}{R_{8}(t) \diamond\left(2-c_{1} c_{2} R_{9}(t)\right)}} \diamond\left(\tanh ^{\diamond}\left(\Delta_{2}(x, y, z, t)\right) \pm i^{\diamond}\left(\Delta_{2}(x, y, z, t)\right)\right) \\
& \pm i \frac{\sqrt{3 b^{* *} c_{2} R_{7}(t)}}{\sqrt{2 R_{8}(t) \diamond R_{9}(t)} \diamond\left(\tanh ^{\diamond}\left(\Delta_{2}(x, y, z, t)\right) \pm i^{\diamond}\left(\Delta_{2}(x, y, z, t)\right)\right)}
\end{aligned}
$$

with

$$
\Delta_{2}(x, y, z, t)=c_{1} x+c_{2} y+c_{3} z-4 c_{1} \int_{0}^{t} \frac{R_{7}(\tau)}{4+c_{1} c_{2} R_{9}(\tau)} d \tau .
$$

(III) Stochastic Travelling Wave Solutions of Trigonometric Type:

$$
\begin{aligned}
W_{4}(x, y, z, t)=W_{5}(x, y, z, t)= & \pm \sqrt{\frac{3 c_{1} c_{2} R_{7}(t)}{1-c_{1} c_{2} R_{8}(t) \diamond R_{9}(t)}} \pm \sqrt{\frac{6 c_{1} c_{2} R_{7}(t) \diamond R_{9}(t)}{1+2 c_{1} c_{2} R_{8}(t) \diamond R_{9}(t)}} \\
& \diamond\left(\sec ^{\diamond}\left(\Delta_{3}(x, y, z, t)\right) \diamond \csc ^{\diamond}\left(\Delta_{3}(x, y, z, t)\right)\right)
\end{aligned}
$$

with

$$
\begin{aligned}
& \Delta_{3}(x, y, z, t)=c_{1} x+c_{2} y+c_{3} z-c_{1} \int_{0}^{t} \frac{R_{7}(\tau)}{1+2 c_{1} c_{2} R_{9}(\tau)} d \tau \\
& W_{6}(x, y, z, t)= \pm 3 \sqrt{\frac{3 c_{1} c_{2} R_{7}(t)}{9 c_{1} c_{2} R_{8}(t) \diamond R_{9}(t)-2}} \pm 4 \sqrt{\frac{3 c_{1} c_{2} R_{7}(t) \diamond R_{9}(t)}{2+15 c_{1} c_{2} R_{8}(t) \diamond R_{9}(t)}} \diamond\left(\cot ^{\diamond}\left(2 \Delta_{4}(x, y, z, t)\right)\right. \\
& \pm i \frac{\sqrt{3 c_{1} R_{7}(t)}}{2 \sqrt{c_{1} R_{8}(t) \diamond R_{9}(t) \diamond\left(\cot ^{\diamond}\left(2 \Delta_{4}(x, y, z, t)\right)\right)}}, \\
& W_{7}(x, y, z, t)= \pm 3 \sqrt{\frac{3 c_{1} c_{2} R_{7}(t)}{9 c_{1} c_{2} R_{8}(t) \diamond R_{9}(t)-2}} \pm 4 \sqrt{\frac{3 c_{1} c_{2} R_{7}(t) \diamond R_{9}(t)}{2+15 c_{1} c_{2} R_{8}(t) \diamond R_{9}(t)}} \diamond\left(\tan ^{\diamond}\left(2 \Delta_{4}(x, y, z, t)\right)\right. \\
& \pm i \frac{\sqrt{3 c_{1} R_{7}(t)}}{2 \sqrt{c_{1} R_{8}(t) \diamond R_{9}(t)} \diamond\left(\tan ^{\diamond}\left(2 \Delta_{4}(x, y, z, t)\right)\right)},
\end{aligned}
$$

with

$$
\Delta_{4}(x, y, z, t)=c_{1} x+c_{2} y+c_{3} z+2 c_{1} \int_{0}^{t} \frac{R_{7}(\tau)}{9 c_{1} c_{2} R_{9}(\tau)-2} d \tau
$$

where $c_{i}(i=1,2,3)$ and $b^{* *}$ are arbitrary constants satisfying $c_{i} b \neq 0$.

\section{Example}

In this section, we provide a specific application example to demonstrate the effectiveness of our results and to justify the real contribution of these results. We focus our attention on Equation (1). Concerning the other two equations, Equations (2) and (3), the procedure is similar. We observe that the solutions of Equation (1) are strongly depend on the shape of the given functions $R_{1}(t)$ and $R_{2}(t)$. So, for dissimilar forms of $R_{1}(t)$ and $R_{2}(t)$, we can find dissimilar solutions of Equation (1) which come from Equations (70)-(78). We illustrate this by giving the following example.

Assume that $R_{2}(t)=\delta_{1} R_{1}(t), R_{3}(t)=\delta_{2} R_{1}(t)$ and $R_{1}(t)=f(t)+\delta_{3} W_{t}$, where $\delta_{1}, \delta_{2}$, and $\delta_{3}$ are arbitrary constants, $f(t)$ is a bounded measurable function on $\mathbb{R}_{+}$, and $W_{t}$ is the Gaussian white noise, which is the time derivative (in the strong sense in $\left.(\mathcal{S})_{-1}\right)$ of the Brownian motion $B_{t}$. The Hermite transform of $W_{t}$ is given by $\widetilde{W}_{t}(w)=\sum_{i=1}^{\infty} w_{i} \int_{0}^{t} \eta_{i}(\tau) d \tau$ [1]. Using the definition of $\widetilde{W}_{t}(w)$, Equations (70)-(78) yield the white noise functional solution of Equation (1) as follows: 


$$
\begin{aligned}
& U_{W_{1}}(x, y, z, t)= \\
& \quad \frac{ \pm i \sqrt{3 a_{3}\left(a_{1} a_{2} \delta_{1} \delta_{2}\left(f(t)+\delta_{3} W_{t}\right)^{2}-2\right)}\left(\exp \left(\Omega_{1}(x, y, z, t)\right)-1\right) \pm \sqrt{3 a_{1} a_{2} a_{3} \delta_{1}}\left(f(t)+\delta_{3} W_{t}\right)}{\sqrt{a_{1} \delta_{1}\left(a_{1} a_{2} \delta_{1} \delta_{2}\left(f(t)+\delta_{3} W_{t}\right)^{2}-2\right)}\left(\exp \left(\Omega_{1}(x, y, z, t)\right)-1\right)}
\end{aligned}
$$

with

$$
\begin{gathered}
\Omega_{1}(x, y, z, t)=a_{1} x+a_{2} y+a_{3} z+2 a_{3} \int_{0}^{t} \frac{f(\tau)+\delta_{3} W_{\tau}}{a_{1} a_{2} \delta_{2}\left(f(\tau)+\delta_{3} W_{\tau}\right)-2} d \tau \\
\begin{aligned}
U_{W_{2}}(x, y, z, t)= & \pm \sqrt{\frac{3 a_{2} a_{3} \delta_{2}\left(f(t)+\delta_{3} W_{t}\right)}{\delta_{1}\left(2-a_{1} a_{2} \delta_{2}\left(f(t)+\delta_{3} W_{t}\right)\right)}}\left(\operatorname{coth}\left(\Omega_{2}(x, y, z, t)\right) \pm\left(\Omega_{2}(x, y, z, t)\right)\right) \\
& \pm i \frac{\sqrt{3 b a_{2}}}{\sqrt{2 \delta_{1} \delta_{2}\left(f(t)+\delta_{3} W_{t}\right)}\left(\operatorname{coth}\left(\Omega_{2}(x, y, z, t)\right) \pm\left(\Omega_{2}(x, y, z, t)\right)\right)} \\
U_{W_{3}}(x, y, z, t)= & \pm \sqrt{\frac{3 a_{2} a_{3} \delta_{2}\left(f(t)+\delta_{3} W_{t}\right)}{\delta_{1}\left(2-a_{1} a_{2} \delta_{2}\left(f(t)+\delta_{3} W_{t}\right)\right)}}\left(\tanh \left(\Omega_{2}(x, y, z, t)\right) \pm i\left(\Omega_{2}(x, y, z, t)\right)\right) \\
& \pm i \frac{\sqrt{3 b a_{2}}}{\sqrt{2 \delta_{1} \delta_{2}\left(f(t)+\delta_{3} W_{t}\right)}\left(\tanh \left(\Omega_{2}(x, y, z, t)\right) \pm i\left(\Omega_{2}(x, y, z, t)\right)\right)}
\end{aligned}
\end{gathered}
$$

with

$$
\begin{gathered}
\Omega_{2}(x, y, z, t)=a_{1} x+a_{2} y+a_{3} z-4 a_{3} \int_{0}^{t} \frac{f(\tau)+\delta_{3} W_{\tau}}{4+a_{1} a_{2} \delta_{2}\left(f(\tau)+\delta_{3} W_{\tau}\right)} d \tau, \\
U_{W_{4}}(x, y, z, t)=U_{W_{5}}(x, y, z, t)= \pm \sqrt{\frac{3 a_{2} a_{3}\left(f(t)+\delta_{3} W_{t}\right)}{1-a_{1} a_{2} \delta_{1} \delta_{2}\left(f(t)+\delta_{3} W_{t}\right)^{2}}} \pm\left(f(t)+\delta_{3} W_{t}\right) \\
\times \sqrt{\frac{6 a_{2} a_{3} \delta_{2}}{1+2 a_{1} a_{2} \delta_{1} \delta_{2}\left(f(t)+\delta_{3} W_{t}\right)^{2}}}\left(\sec \left(\Omega_{3}(x, y, z, t)\right) \csc \left(\Omega_{3}(x, y, z, t)\right)\right),
\end{gathered}
$$

with

$$
\begin{aligned}
\Omega_{3}(x, y, z, t)= & a_{1} x+a_{2} y+a_{3} z-a_{3} \int_{0}^{t} \frac{f(\tau)+\delta_{3} W_{\tau}}{1+2 a_{1} a_{2} \delta_{2}\left(f(\tau)+\delta_{3} W_{\tau}\right)} d \tau, \\
U_{W_{6}}(x, y, z, t)= & \pm 3 \sqrt{\frac{3 a_{2} a_{3}\left(f(t)+\delta_{3} W_{t}\right)}{9 a_{1} a_{2} \delta_{1} \delta_{2}\left(f(t)+\delta_{3} W_{t}\right)^{2}-2}} \pm 4\left(f(t)+\delta_{3} W_{t}\right) \\
& \times \sqrt{\frac{3 a_{2} a_{3} \delta_{2}}{2+15 a_{1} a_{2} \delta_{1} \delta_{2}\left(f(t)+\delta_{3} W_{t}\right)^{2}}}\left(\cot \left(2 \Omega_{4}(x, y, z, t)\right)\right) \\
& \pm i \frac{\sqrt{3 a_{3}}}{2 \sqrt{a_{1} \delta_{1} \delta_{2}\left(\cot \left(2 \Omega_{4}(x, y, z, t)\right)\right)}} \\
U_{W_{6}}(x, y, z, t)= & \pm 3 \sqrt{\frac{3 a_{2} a_{3}\left(f(t)+\delta_{3} W_{t}\right)}{9 a_{1} a_{2} \delta_{1} \delta_{2}\left(f(t)+\delta_{3} W_{t}\right)^{2}-2}} \pm 4\left(f(t)+\delta_{3} W_{t}\right) \\
& \times \sqrt{\frac{3 a_{2} a_{3} \delta_{2}}{2+15 a_{1} a_{2} \delta_{1} \delta_{2}\left(f(t)+\delta_{3} W_{t}\right)^{2}}}\left(\tan \left(2 \Omega_{4}(x, y, z, t)\right)\right) \\
& \pm i \frac{\sqrt{3 a_{3}}}{2 \sqrt{a_{1} \delta_{1} \delta_{2}}\left(\tan \left(2 \Omega_{4}(x, y, z, t)\right)\right)}
\end{aligned}
$$


with

$$
\Omega_{4}(x, y, z, t)=a_{1} x+a_{2} y+a_{3} z+2 a_{3} \int_{0}^{t} \frac{f(\tau)+\delta_{3} W_{\tau}}{9 a_{1} a_{2} \delta_{2}\left(f(\tau)+\delta_{3} W_{\tau}\right)-2} d \tau
$$

\section{Conclusions}

Due to the fact that the stochastic models are more realistic than the deterministic models, we concentrated our study in this paper on the Wick-type, stochastic, $(3+1)$-dimensional modified BBM equations. Besides that, we investigated and solve the deterministic, $(3+1)$-dimensional modified BBM equations. In this paper, we set up a new and general version of the modified tanh-coth method to deal with the nonlinear multi dimensional PDEs. By using this generalisation of the modified tanh-coth method, Hermite transform, and white noise theory, we produced a new set of exact travelling wave solutions for the variable coefficients and (3+1)-dimensional modified BBM equations. This set includes solutions of exponential, hyperbolic, and trigonometric types. In [7], Wazwaz has solved the deterministic, (3+1)-dimensional modified BBM equations with constant coefficients, So, our results for this model are more general than the results obtained by him. With the aid of inverse Hermite transform, we obtained stochastic travelling wave solutions for the Wick-type, stochastic, (3+1)-dimensional modified BBM equations. Furthermore, we showed by an example how the stochastic solutions can be given as white noise functional solutions. Note that, the schema proposed in this paper can be used for solving several nonlinear evolution equations in mathematical physics, both Wick-type stochastic and deterministic. Moreover, the Riccati equation (19) has different solutions if we chose different values of $\alpha_{0}, \alpha_{1}$, and $\alpha_{2}$. Therefore, we can find many other solutions of the Wick-type stochastic and deterministic $(3+1)$-dimensional modified BBM equations.

The PDE of Benjamin et al. [2] is now often called the BBM equation, although it is also known as the regularised long wave (RLW) equation. Morrison et al. proposed the one-dimensional PDE, as an equally valid and accurate model for the same wave phenomena simulated by the KdV and RLW equations [40]. Random waves are an important subject of random PDEs. In essence, to investigate the exact solutions of random Benjamin-Bona-Mahony equation, we restricted our attention to consider this problem in a white noise environment [38]. The propagation of nonlinear wave in systems with polarity symmetry can be described by the (3+1)-dimensional modified Benjamin-Bona-Mahony Equation (7). If the problem is considered in a non-Gaussian stochastic environment, we can get non-Gaussian, stochastic, (2+1)-dimensional coupled KdV equation. Obviously, the planner which we have proposed in this paper can be also applied to other non-linear PDEs in mathematical physics such as KdV-Burgers, modified KdV-Burgers, Zhiber-Shabat and Benjamin-Bona-Mahony equations. We observe that the F-expansion method we used has many other particular solutions; this in turn gives many other exact solutions for the considered stochastic, $(3+1)$-dimensional modified Benjamin-Bona-Mahony equations. Additionally, in this work, we discussed the solutions of SPDEs driven by non-Gaussian white noise; this discussion is less detailed than the Gaussian discussion but more general, because it deals with the dual pairing generated by integration with respect to a non-Gaussian measure. Furthermore, in future work, we will discuss the solutions of SPDEs driven by non-Gaussian white noise to get exact stochastic solutions of the non-Gaussian, stochastic, (3+1)-dimensional modified Benjamin-Bona-Mahony equations; we only considered this problem in a non-Gaussian white noise environment; that is, we investigated the variable coefficients of stochastic, (3+1)-dimensional modified Benjamin-Bona-Mahony equations. For this aim, we developed a non-Gaussian Wick calculus based on the theory of hyper-complex systems to get exact travelling wave solutions of (3+1)-dimensional modified Benjamin-Bona-Mahony equations and non-Gaussian white noise functional solutions of Wick-type stochastic (3+1)-dimensional modified Benjamin-Bona-Mahony equations.

Author Contributions: The authors have equally contributed to the research.

Funding: This research was funded by the Deanship of Scientific Research at Princess Nourah bint Abdulrahman University through the Fast-track Research Funding Program. 
Conflicts of Interest: The authors declare no conflict of interest.

\section{References}

1. Holden, H.; Øsendal, B.; Ubøe, J.; Zhang, T. Stochastic Partial Differential Equations; Springer Science+Business Media, LLC: New York, NY, USA, 2010.

2. Benjamin, T.B.; Bona, J.L.; Mahony, J.J. Model equations for long waves in nonlinear dispersive systems. Trans. R. Soc. Lond. Ser. A 1972, 272, 47-78. [CrossRef]

3. Dehghan, M.; Shokri, A. A numerical method for solution of the two-dimensional sine-Gordon equation using the radial basis functions. Comput. Math. Simul. 2008, 79, 700-715. [CrossRef]

4. Hereman, W. Exact Solutions of Nonlinear Partial Differential Equations. The tanh/sech Method; Wolfram Research Academic Intern Program Inc.: Champaign, IL, USA, 2000; pp. 1-14.

5. Hereman, W. Shallow water waves and solitary waves. In Encyclopedia of Complexity and Systems Science; Meryers, R.A., Ed.; Springer: Heibelberg, Germany, 2009; pp. 1536-1620.

6. Khalique, C.M. Exact solutions and conservation laws of a coupled integrable dispersionless system. Filomat 2012, 26, 957-964. [CrossRef]

7. Wazwaz, A.M. Exact soliton and kink solutions for new (3+1)-dimensional nonlinear modified equations of wave propagation. Open Eng. 2017, 7, 169-174. [CrossRef]

8. Dai, C.Q.; Xu, Y.J. Exact solutions for a Wick-type stochastic reaction Duffing equation. Appl. Math. Model. 2015, 39, 7420-7426. [CrossRef]

9. Kong, L.Q.; Dai, C.Q. Some discussions about variable separation of nonlinear models using Riccati equation expansion method. Nonlinear Dyn. 2015, 81, 1553-1561. [CrossRef]

10. Wang, Y.Y.; Zhang, Y.P.; Dai, C.Q. Re-study on localized structures based on variable separation solutions from the modified tanh-function method. Nonlinear Dyn. 2015, 83, 1331-1339. [CrossRef]

11. Wadati, M. Stochastic Korteweg-de Vries equation. J. Phys. Soc. Jpn. 1983, 52, 2642-2648. [CrossRef]

12. Ghany, H.A.; Hyder, A. White noise functional solutions for the Wick-type two dimensional stochastic Zakharov-Kuznetsov equations. Int. Rev. Phys. 2012, 6, 153-157.

13. Ghany, H.A.; Hyder, A. The fractional coupled KdV equations: Exact solutions and white noise functional approach. Chin. Phys. B 2013, 22, 0805011. [CrossRef]

14. Ghany, H.A.; Hyder, A. Exact solutions for the Wick-type stochastic time-fractional KdV equations. Kuwait J. Sci. 2014, 41, 75-84.

15. Ghany, H.A.; Hyder, A. Abundant solutions of Wick-type stochastic fractional 2D KdV equations. Chin. Phys. B 2014, 23, 0605031. [CrossRef]

16. Ghany, H.A.; Hyder, A.; Zakarya, M. Non-gaussian white noise functional solutions of $\chi$-Wick-type stochastic KdV equations. Appl. Math. Inf. Sci. 2017, 11, 915-924. [CrossRef]

17. Ghany, H.A.; Hyder, A.; Zakarya, M. Exact Solutions of Stochastic Fractional Korteweg de-Vries Equation with Conformable Derivatives. Chin. Phys. B 2019, 28, 191092.

18. Chen, B.; Xie, Y.C. Exact solutions for generalized stochastic Wick- type KdV-mKdV equations. Chaos Solitons Fractals 2005, 23, 281-288. [CrossRef]

19. Chen, B.; Xie, Y.C. White noise functional solutions of Wick-type stochastic generalized Hirota-Satsuma coupled KdV equations. J. Comput. Appl. Math. 2006, 157, 345-354. [CrossRef]

20. Chen, B.; Xie, Y.C. Periodic-like solutions of variable coefficient and Wick-type stochastic NLS equations. J. Comput. Appl. Math. 2007, 203, 249-263. [CrossRef]

21. Hyder, A.; Zakarya, M. Non-Gaussian Wick calculus based on hypercomplex systems. Int. J. Pure Appl. Math. 2016, 109, 539-556. [CrossRef]

22. Hyder, A.; Zakarya, M. The Well-Posedness of Stochastic Kawahara Equation: Fixed Point Argument and Fourier Restriction Method. J. Egypt. Math. Soc. 2019, 27, 1-10.

23. Hyder, A. Wick-type stochastic KdV equation based on Lévy white noise. J. Math. Sci. Adv. Appl. 2017, 45, 1-20. [CrossRef]

24. Hyder, A. White noise analysis combined with hypercomplex systems for solving stochastic modified KdV equations with non-Gaussian parameters. Pioneer J. Adv. Appl. Math. 2018, 24, 39-61.

25. Agarwal, P.; Hyder, A.; Zakarya, M. Well-Posedness of Stochastic Modified Kawahara Equation. Adv. Differ. Equ. 2019, 2019, 423. 
26. Liu, X.Q.; Jiang, S.; Fan, W.B.; Liu, W.M. Soliton solutions in linear magnetic field and time-dependent laser field. Commun. Nonlinear Sci. Numer. Simul. 2004, 9, 361-365. [CrossRef]

27. Ivaz, K.; Mostahkam, B.S. Newton-Tau numerical solution of a system of nonlinear Fredholm integral equations of second kind. Appl. Comput. Math. 2006, 5, 201-208.

28. Wazwaz, A.M. New solitons and kinks solutions for the Gardner equation. Commun. Nonlinear Sci. Numer. Simul. 2007, 12, 1395-1404. [CrossRef]

29. Pourgholi, R.; Esfahani, A.; Houlari, T.; Foadian, S. An application of Sinc-Galerkin method for solving the Tzou equation. Appl. Comput. Math. 2017, 16, 257-268.

30. Momani, S.; Arqub, O.A.; Maayah, B.; Yousef, F.; Alsaedi, A. A reliable algorithm for solving linear and nonlinear Schrödinger equation. Appl. Comput. Math. 2018, 17, 151-160.

31. El-Ganaini, S.I.A.; Mirzazadeh, M.; Biswas, A. Solitons and other solutions to long-short wave resonance equation. Appl. Comput. Math. 2015, 14, 248-259.

32. Bhalekar, S.; Patade, J. Analytical solutions of nonlinear equations with proportional delays. Appl. Comput. Math. 2016, 15, 331-345.

33. Malfeit, W. Solitary wave solutions of nonlinear wave equations. Am. J. Phys. 1992, 60, 650-654.

34. Fan, E.G. Extended tanh-function method and its applications to nonlinear equations. Phys. Lett. A 2000, 277, 212-218. [CrossRef]

35. Wazwaz, A.M. The tanh-coth method for solitons and kink solutions for nonlinear parabolic equations. Appl. Math. Comput. 2007, 188, 1467-1475. [CrossRef]

36. El-Wakil, S.A.; El-Labany, S.K.; Zahran, M.A.; Sabry, R. Modified extended tanh-function method and its applications to nonlinear equations. Appl. Math. Comput. 2005, 161, 403-412.

37. Soliman, A.A. The modified extended tanh-function method for solving Burgers-type equations. Phys. A 2006, 361, 394-404. [CrossRef]

38. Ghany, H.A.; Fathallah, A. White-noise functional solutions for wick-type stochastic time-fractional Benjamin-Bona-Mahony equation. Int. J. Diff. Equ. Appl. 2014, 13, 37-49.

39. Sahoo, S.; Saha Ray, S.A. Novel approach for stochastic solutions of wick-type stochastic time-fractional Benjamin-Bona-Mahony equation for modeling long surface gravity waves of small amplitude. Stoch. Anal. Appl. 2019, 2019, 1-12. [CrossRef]

40. Korteweg, D.J.; de Vries, G. On the change of form of long waves advancing in a rectangular canal, and on a new type of long stationary waves. Phil. Mag. Ser. 1895, 39, 422-443. [CrossRef]

41. Larin, V.B. High-accuracy algorithms for solution of discrete periodic Riccati equations. Appl. Comput. Math. 2007, 6, 10-17.

42. Chen, H.; Zhang, H. New multiple soliton-like solutions to the generalized (2+1)-dimensional KP equation. Appl. Math. Comput. 2004, 157, 765-773. [CrossRef] 